\title{
9. GRAIN-SIZE DISTRIBUTION FROM THE SOUTHEASTERN TIP OF BAJA CALIFORNIA AND GUAYMAS BASIN, MEXICO, DEEP SEA DRILLING PROJECT LEG $64^{1}$
}

\author{
M. Gutiérrez-Estrada, ${ }^{2}$ Universidad Naciónal Autónoma de Mexico, \\ Centro de Ciencias del mar y Limnologia, Mexico City, Mexico
}

\begin{abstract}
Grain size of 139 unconsolidated sediment samples from seven DSDP sites in the Guaymas Basin and the southeastern tip of the Baja California Peninsula was determined by sieve and pipette techniques. Shepard (1954) classification and Inman (1952) parameters correlation were used for all samples. Sediment texture ranged from sand to silty clay. On the basis of grain-size parameter, the sediments can be divided into three broad groups: (1) very fine sands and coarse silts; (2) medium- to very fine silts; and (3) clays and coarse silts.
\end{abstract}

\section{INTRODUCTION}

This chapter presents information on grain size from Pleistocene and Pliocene deep-sea sediments cored at seven sites in the Guaymas Basin and the southeastern tip of Baja California (Fig. 1). Grain size is a fundamental property of deep-sea sediments and sedimentary rock (Griffiths, 1967; Blatt et al., 1972). According to Thayer et al. (1974), little detailed work on grain-size distribution or statistical parameters has been done. This chapter provides basic data on grain size for different lithological units cored at these sites. The information may be useful for relating size distribution to environments of deposition, physical mechanisms of sedimentation, and physical properties of the sediments. Site locations are indicated in Figure 1.

\section{METHODS}

The distribution of sand-silt-clay was determined with $10 \mathrm{~cm}^{3}$ sediment samples collected when the cores were split and described (see Tables 1, 2). The sediment classification (Fig. 2) is Shepard's (1954), with sand, silt, and clay boundaries based on the Wentworth (1922) scale. The parameters correlation (Fig. 3) was suggested by Inman (1952). Sand, silt, and clay fractions contain particles whose diameters range from 2000 to $62.5 \mu \mathrm{m}, 62.5$ to $3.91 \mu \mathrm{m}$, and less than $3.91 \mu \mathrm{m}$, respectively. This classification is applied regardless of sediment type and origin (Bode, 1974), hence the sediment names used in Table 1 may differ from those used elsewhere in this volume.

Standard sieve, settling tube, and pipette methods determined the grain-size distribution. The samples were dried and dispersed by soaking for 24 hours in $200 \mathrm{ml}$ of distilled water. If a sample failed to disaggregate, it was treated with hydrogen peroxide and $50 \mathrm{ml}$ of $10 \%$ sodium hexametaphosphate (calgon). If lumps of mud remained after soaking, they were removed by gentle crushing with a rubber-gloved finger (Folk, 1968).

The sand fraction was removed by wet sieving using a $62.5 \mu \mathrm{m}$ sieve (No. 230 U.S. Standard Sieve) to separate it from the mud (silt and clay) fraction. The silt and clay fractions were analyzed by pipette analysis (Folk, 1968). Sampling depths and times were calculated using equations derived from Stokes's settling velocity equation (Krumbein and Pettijohn, 1938). Pipette withdrawals were taken at times corresponding to $1.0 \phi$ unit intervals from $4.0 \phi$ to $11.0 \phi$. The pipette analyses were terminated at $11.0 \phi$, because particles smaller than this

${ }^{1}$ Curray, J. R., Moore, D. G., et al., Init. Repts. DSDP, 64: Washington (U.S. Govt. Printing Office).

2 Present address: Estación de Investigaciones Mazatlán, Universidad Naciónal Autónoma de Mexico, Mazatlán, Sinaloa, Mexico.

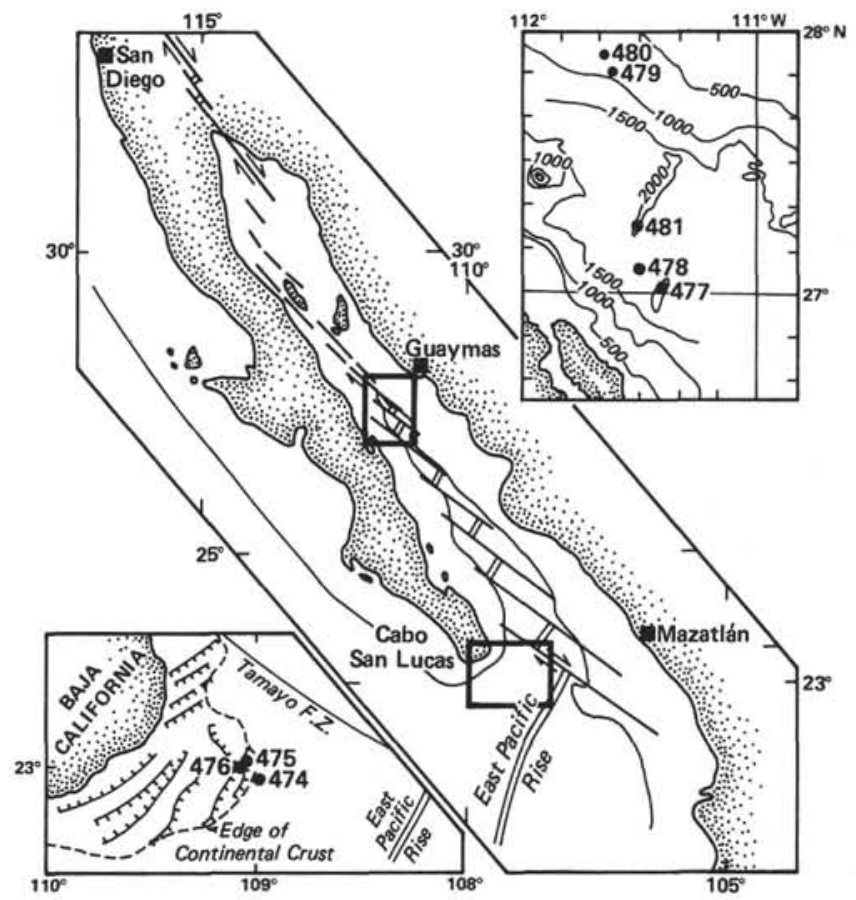

Figure 1. Leg 64 drilling sites.

diameter are strongly affected by Brownian movement (Irani and Callis, 1963; Thayer et al., 1974). The sand fraction was dried and analyzed at $1 / 4 \phi$ and $1 / 2 \phi$ by settling tube method (Emery, 1938).

Cumulative percentages of sand and mud were determined, and cumulative curves were drawn on probability graph paper (probability scale $\times 90$ division). If the cumulative percentage at $11.0 \phi$ was less than $95 \%$, the unsampled fine population was interpolated by extending the cumulative curve in a line from $11.0 \phi$ to $14.0 \phi$ at $100 \%$ (Folk, 1968). This operation assumes that all sediment is coarser than $14.0 \phi$, and that the clay mode lies near $12.0 \phi$. Cumulative percentage values for $12.0 \phi$ and $13.0 \phi$ were read directly from the interpolated curve (Thayer, et al., 1974).

Folk and Ward (1957) and Inman (1952) statistics were calculated (Table 2). Folk and Ward (1957) statistics are used, since they are the most widely used by sedimentologists. The Folk and Ward (1957) measures are given in the following formulas:

Graphic mean:

$$
M_{Z}=\frac{\phi 16+\phi 50+\phi 84 .}{3} \text {. }
$$


Table 1. Folk's statistical parameters.

\begin{tabular}{|c|c|c|c|c|c|c|c|}
\hline $\begin{array}{c}\text { Sample } \\
\text { (interval in } \mathrm{cm} \text { ) }\end{array}$ & $\begin{array}{l}\text { Depth } \\
\text { (m) }\end{array}$ & $\phi 5$ & $M_{Z}(\phi)$ & $\sigma_{I}(\phi)$ & $S K_{I}$ & $K_{G}$ & $K^{\prime} G$ \\
\hline \multicolumn{8}{|l|}{ Hole 474} \\
\hline $1-2,45-47$ & 1.95 & 4.00 & 8.700 & 2.487 & 0.038 & 0.771 & 0.435 \\
\hline $2-2,55-57$ & 4.05 & 3.75 & 7.200 & 2.691 & 0.053 & 0.709 & 0.415 \\
\hline $3-2,50-52$ & 13.50 & 4.40 & 7.866 & 2.263 & -0.072 & 1.020 & 0.505 \\
\hline $4-2,80-82$ & 23.30 & 4.20 & 8.800 & 2.366 & -0.098 & 0.956 & 0.489 \\
\hline $5-3,47-49$ & 33.30 & 3.05 & 7.466 & 2.792 & -0.163 & 0.865 & 0.464 \\
\hline $6-4,69-71$ & 45.19 & 3.00 & 6.700 & 2.662 & 0.043 & 0.728 & 0.421 \\
\hline $7-5,110-112$ & 56.60 & 1.15 & 4.000 & 2.486 & 0.600 & 1.358 & 0.576 \\
\hline $8-2,52-54$ & 61.02 & 0.70 & 1.886 & 1.363 & 0.319 & 1.467 & 0.594 \\
\hline $11-2,114-116$ & 90.14 & 2.60 & 7.400 & 2.186 & -0.524 & 1.414 & 0.585 \\
\hline $12-1,40-42$ & 97.40 & 4.75 & 8.783 & 2.320 & -0.027 & 0.741 & 0.426 \\
\hline $13-1,49-51$ & 106.99 & 4.50 & 7.433 & 2.361 & 0.387 & 0.842 & 0.457 \\
\hline $14-2,47-49$ & 117.97 & 4.30 & 8.533 & 2.426 & 0.042 & 0.819 & 0.450 \\
\hline $16-3,0-2$ & 138.00 & 3.85 & 7.066 & 2.230 & 0.051 & 0.875 & 0.467 \\
\hline $16-3,9-11$ & 138.09 & 0.00 & 2.183 & 1.509 & 0.004 & 1.639 & 0.621 \\
\hline $17-5,25-27$ & 150.75 & 3.90 & 7.850 & 2.323 & -0.222 & 0.914 & 0.477 \\
\hline $17-5,57-57$ & 151.05 & 4.20 & 8.416 & 2.574 & -0.017 & 0.756 & 0.430 \\
\hline $17-5,77-79$ & 151.27 & 3.00 & 6.886 & 2.958 & 0.234 & 0.770 & 0.435 \\
\hline $18-3,32-34$ & 157.32 & 3.20 & 7.466 & 2.654 & -0.091 & 0.850 & 0.459 \\
\hline $19-2,90-92$ & 165.90 & 3.90 & 7.750 & 2.163 & -0.212 & 1.058 & 0.514 \\
\hline \multicolumn{8}{|l|}{ Hole 474A } \\
\hline $1-4,68-70$ & 168.68 & 4.20 & 7.983 & 2.431 & -0.130 & 0.798 & 0.444 \\
\hline $1-4,85-87$ & 168.85 & 5.15 & 8.073 & 2.283 & 0.272 & 0.913 & 0.477 \\
\hline $1-4,101-103$ & 169.01 & 3.01 & 4.366 & 1.632 & 0.730 & 2.584 & 0.721 \\
\hline $2-1,103-105$ & 174.03 & 3.42 & 5.210 & 1.741 & 0.492 & 1.608 & 0.616 \\
\hline $2-1,137-139$ & 174.37 & 2.90 & 4.960 & 2.460 & 0.734 & 1.710 & 0.631 \\
\hline $3-5,35-37$ & 188.84 & 4.75 & 7.420 & 2.122 & 0.324 & 0.845 & 0.460 \\
\hline $3-5,78-80$ & 189.28 & 4.50 & 7.316 & 2.140 & -0.291 & 0.900 & 0.473 \\
\hline $3-5,109-111$ & 189.59 & 2.50 & 4.583 & 1.940 & 0.471 & 0.912 & 0.477 \\
\hline $3-5,137-139$ & 189.87 & 2.86 & 3.840 & 1.080 & 0.611 & 2.843 & 0.740 \\
\hline $4-4,76-78$ & 197.26 & 4.80 & 8.500 & 2.023 & -0.055 & 1.082 & 0.520 \\
\hline $6-1,99-101$ & 211.99 & 4.65 & 7.533 & 2.181 & 0.227 & 0.917 & 0.480 \\
\hline $6-1,148-150$ & 212.48 & 3.15 & 5.320 & 2.384 & 0.664 & 1.491 & 0.598 \\
\hline $6-2,11-13$ & 212.61 & 1.06 & 5.633 & 3.376 & -0.058 & 0.684 & 0.406 \\
\hline $7-2,49-51$ & 222.49 & 3.90 & 8.000 & 2.767 & 0.331 & 0.613 & 0.380 \\
\hline $9-3,59-60$ & 243.09 & 4.37 & 7.433 & 2.275 & 0.253 & 0.835 & 0.455 \\
\hline $10-2,60-62$ & 251.10 & 4.32 & 6.916 & 1.971 & 0.107 & 0.803 & 0.445 \\
\hline $12-3,62-64$ & 271.62 & 4.08 & 6.800 & 1.678 & 0.071 & 0.764 & 0.433 \\
\hline $13-3,81-83$ & 281.31 & 5.35 & 7.710 & 1.942 & 0.322 & 0.929 & 0.481 \\
\hline $13-4,7-9$ & 282.81 & 4.26 & 7.150 & 2.190 & 0.338 & 1.053 & 0.513 \\
\hline $13-4,108-110$ & 283.08 & 2.86 & 5.623 & 2.068 & 0.446 & 1.062 & 0.515 \\
\hline $13-4,116-118$ & 283.16 & 3.76 & 6.233 & 1.967 & 0.104 & 0.680 & 0.404 \\
\hline $16-3,67-69$ & 309.67 & 4.00 & 7.450 & 2.598 & 0.293 & 0.768 & 0.434 \\
\hline $17-4,82-84$ & 320.82 & 4.55 & 6.983 & 1.911 & 0.155 & 1.042 & 0.510 \\
\hline $19-2,52-54$ & 329.02 & 4.65 & 7.816 & 2.222 & 0.061 & 0.907 & 0.475 \\
\hline $21-5,79-81$ & 350.79 & 4.40 & 7.633 & 2.393 & 0.055 & 0.780 & 0.438 \\
\hline $23-1,88-90$ & 363.88 & 1.50 & 5.316 & 2.697 & 0.116 & 0.781 & 0.438 \\
\hline $23-4,87-89$ & 368.37 & 4.43 & 7.513 & 2.443 & 0.197 & 0.738 & 0.424 \\
\hline $23-4,113-115$ & 368.63 & 4.14 & 7.050 & 2.415 & 0.401 & 0.887 & 0.470 \\
\hline $25-1,62-64$ & 382.62 & 4.30 & 7.016 & 1.793 & 0.195 & 0.915 & 0.478 \\
\hline $25-4,142-144$ & 387.92 & 4.90 & 7.100 & 1.653 & 0.210 & 0.841 & 0.457 \\
\hline $25-5, C C, 16-18$ & 388.68 & 3.80 & 6.400 & 2.286 & 0.337 & 1.007 & 0.501 \\
\hline $27-4,81-83$ & 406.31 & 4.15 & 6.653 & 2.081 & 0.653 & 1.516 & 0.602 \\
\hline $28-4,69-71$ & 414.69 & 4.86 & 8.416 & 2.146 & 0.004 & 0.812 & 0.448 \\
\hline $29-2,112-114$ & 422.62 & 4.71 & 6.600 & 1.664 & -0.645 & 1.248 & 0.555 \\
\hline $30-1,121-123$ & 430.71 & 4.27 & 6.460 & 2.002 & 0.505 & 1.142 & 0.533 \\
\hline $30-2,3-5$ & 431.03 & 3.80 & 6.183 & 2.014 & 0.234 & 0.833 & 0.454 \\
\hline $30-2,43-45$ & 431.43 & 3.20 & 5.633 & 2.293 & 0.468 & 0.910 & 0.476 \\
\hline $30-2,113-115$ & 432.13 & 1.66 & 3.793 & 2.101 & 0.650 & 1.324 & 0.569 \\
\hline $30-3,68-70$ & 433.18 & 4.50 & 8.133 & 2.663 & -0.065 & 0.627 & 0.385 \\
\hline $31-3,54-56$ & 442.53 & 4.70 & 6.716 & 1.673 & 0.334 & 0.868 & 0.464 \\
\hline $32-5,108-110$ & 455.58 & 4.30 & 6.316 & 2.023 & 0.540 & 1.195 & 0.544 \\
\hline $33-3,83-85$ & 460.83 & 3.96 & 6.950 & 2.072 & 0.058 & 0.745 & 0.426 \\
\hline $36-2,104-106$ & 489.04 & 4.60 & 7.666 & 1.941 & -0.130 & 0.826 & 0.452 \\
\hline \multicolumn{8}{|l|}{ Hole 475} \\
\hline $1-2,62-64$ & 2.12 & 4.50 & 8.546 & 2.131 & -0.316 & 1.004 & 0.501 \\
\hline $2-5,121-123$ & 12.62 & 2.50 & 5.583 & 3.291 & 0.673 & 0.656 & 0.396 \\
\hline $3-2,74-76$ & 17.74 & 4.25 & 7.300 & 1.790 & -0.509 & 0.988 & 0.497 \\
\hline $5-4,71-73$ & 39.71 & 4.90 & 8.250 & 2.553 & -0.021 & 0.619 & 0.382 \\
\hline $6-5,23-25$ & 50.23 & 4.00 & 7.133 & 1.859 & -0.396 & 1.069 & 0.516 \\
\hline $8-5,64-66$ & 69.64 & 4.20 & 7.683 & 2.476 & 0.060 & 0.987 & 0.496 \\
\hline $9-4,67-69$ & 77.67 & 4.20 & 7.966 & 2.325 & -0.265 & 0.786 & 0.440 \\
\hline $11-6,16-18$ & 99.16 & 4.10 & 7.500 & 1.896 & -0.469 & 0.785 & 0.439 \\
\hline $12-5,31-33$ & 107.31 & 4.50 & 6.800 & 1.394 & -0.214 & 0.949 & 0.487 \\
\hline $13-4,51-53$ & 115.51 & 4.60 & 7.933 & 2.528 & 0.270 & 0.719 & 0.418 \\
\hline $15-2,80-82$ & 131.80 & 3.90 & 7.833 & 2.663 & 0.116 & 1.053 & 0.513 \\
\hline $15-3,77-79$ & 133.27 & 2.65 & 4.666 & 2.325 & 0.748 & 1.668 & 0.625 \\
\hline
\end{tabular}

Table 1. (Continued).

\begin{tabular}{|c|c|c|c|c|c|c|c|}
\hline $\begin{array}{c}\text { Sample } \\
\text { (interval in } \mathrm{cm} \text { ) }\end{array}$ & $\begin{array}{l}\text { Depth } \\
\text { (m) }\end{array}$ & $\phi 5$ & $M_{Z}(\phi)$ & $\sigma_{I}(\phi)$ & $S K_{I}$ & $K_{G}$ & $K^{\prime} G$ \\
\hline \multicolumn{8}{|l|}{ Hole 475 (Cont.) } \\
\hline $\begin{array}{l}15-3,99-101 \\
15-4,104-106 \\
15-4,122-124 \\
15-6,65-67 \\
16-5,33-35 \\
17-2,44-46 \\
17-4,42-44\end{array}$ & $\begin{array}{l}133.49 \\
135.04 \\
135.22 \\
137.65 \\
145.33 \\
150.44 \\
153.42\end{array}$ & $\begin{array}{l}4.35 \\
3.80 \\
3.60 \\
4.15 \\
4.40 \\
3.75 \\
4.60\end{array}$ & $\begin{array}{l}6.966 \\
7.000 \\
6.210 \\
7.866 \\
6.933 \\
6.600 \\
7.316\end{array}$ & $\begin{array}{l}1.876 \\
2.140 \\
2.112 \\
2.690 \\
2.075 \\
2.333 \\
2.062\end{array}$ & $\begin{array}{l}0.207 \\
0.070 \\
0.307 \\
0.184 \\
0.332 \\
0.189 \\
0.248\end{array}$ & $\begin{array}{l}0.920 \\
0.858 \\
0.872 \\
1.209 \\
1.001 \\
0.901 \\
1.001\end{array}$ & $\begin{array}{l}0.479 \\
0.461 \\
0.465 \\
0.547 \\
0.500 \\
0.474 \\
0.500\end{array}$ \\
\hline \multicolumn{8}{|l|}{ Hole 475B } \\
\hline $1-7,61-63$ & 9.61 & 4.50 & 0.033 & 1.709 & -0.289 & 1.439 & 0.590 \\
\hline \multicolumn{8}{|l|}{ Hole 476} \\
\hline $\begin{array}{l}1-2,48-50 \\
2-4,130-132 \\
3-3,89-91 \\
5-3,77-79 \\
6-4,71-73 \\
7-4,106-108 \\
8-2,126-128 \\
10-4,57-59 \\
11-6,99-101 \\
13-5,65-67 \\
15-3,77-79 \\
16-3,67-69 \\
17-1,85-87 \\
18-5,65-67 \\
19-1,86-88 \\
20-5,69-71 \\
21-3,28-30\end{array}$ & $\begin{array}{r}1.98 \\
14.80 \\
22.39 \\
41.27 \\
53.21 \\
61.71 \\
68.76 \\
90.07 \\
102.99 \\
120.15 \\
136.27 \\
145.67 \\
152.35 \\
167.65 \\
171.36 \\
186.69 \\
192.78\end{array}$ & $\begin{array}{l}2.10 \\
3.35 \\
4.50 \\
2.65 \\
3.24 \\
4.70 \\
4.70 \\
5.05 \\
4.30 \\
4.15 \\
1.65 \\
2.55 \\
4.37 \\
3.80 \\
3.85 \\
3.05 \\
1.70\end{array}$ & $\begin{array}{l}8.133 \\
7.463 \\
8.700 \\
6.116 \\
6.333 \\
8.133 \\
8.616 \\
8.6931 \\
8.550 \\
7.133 \\
4.216 \\
7.483 \\
7.770 \\
8.000 \\
6.600 \\
6.900 \\
5.166\end{array}$ & $\begin{array}{l}3.133 \\
2.401 \\
2.408 \\
2.746 \\
2.264 \\
2.403 \\
2.215 \\
2.309 \\
2.489 \\
1.756 \\
2.030 \\
3.268 \\
2.668 \\
2.381 \\
1.915 \\
2.714 \\
3.256\end{array}$ & $\begin{array}{r}-0.133 \\
-0.054 \\
-0.046 \\
0.122 \\
-0.026 \\
-0.062 \\
-0.102 \\
0.010 \\
-0.304 \\
-0.087 \\
0.463 \\
-0.118 \\
0.088 \\
0.004 \\
0.089 \\
-0.069 \\
0.712\end{array}$ & $\begin{array}{l}0.817 \\
0.966 \\
0.739 \\
0.616 \\
0.617 \\
0.780 \\
0.863 \\
0.720 \\
0.750 \\
1.028 \\
1.223 \\
0.819 \\
0.711 \\
1.175 \\
1.339 \\
0.873 \\
1.010\end{array}$ & $\begin{array}{l}0.449 \\
0.491 \\
0.425 \\
0.381 \\
0.381 \\
0.438 \\
0.463 \\
0.418 \\
0.428 \\
0.507 \\
0.550 \\
0.450 \\
0.415 \\
0.540 \\
0.572 \\
0.466 \\
0.502\end{array}$ \\
\hline \multicolumn{8}{|l|}{ Hole 477} \\
\hline $\begin{array}{l}3-2,8-10 \\
3-2,113-115 \\
4-1,81-83 \\
5-1,78-80 \\
7-1,22-24 \\
15-1,11-13 \\
16-2,24-26 \\
17-3,21-23 \\
19-2,135-137 \\
20-2,84-86\end{array}$ & $\begin{array}{r}12.08 \\
13.13 \\
20.81 \\
30.28 \\
48.72 \\
105.11 \\
116.74 \\
127.71 \\
146.35 \\
155.34\end{array}$ & $\begin{array}{l}5.75 \\
4.35 \\
4.85 \\
3.90 \\
4.50 \\
4.80 \\
4.60 \\
4.00 \\
2.85 \\
5.00\end{array}$ & $\begin{array}{l}9.133 \\
8.550 \\
8.716 \\
7.916 \\
7.450 \\
8.233 \\
8.000 \\
7.933 \\
6.060 \\
7.766\end{array}$ & $\begin{array}{l}2.021 \\
2.537 \\
2.468 \\
2.676 \\
1.423 \\
2.370 \\
2.508 \\
2.694 \\
2.960 \\
2.490\end{array}$ & $\begin{array}{r}0.080 \\
0.017 \\
-0.062 \\
0.108 \\
-0.171 \\
0.102 \\
0.225 \\
0.000 \\
0.577 \\
0.315\end{array}$ & $\begin{array}{l}0.711 \\
0.860 \\
0.713 \\
0.804 \\
1.289 \\
0.856 \\
0.837 \\
0.781 \\
0.848 \\
0.713\end{array}$ & $\begin{array}{l}0.415 \\
0.462 \\
0.416 \\
0.445 \\
0.563 \\
0.461 \\
0.455 \\
0.438 \\
0.459 \\
0.416\end{array}$ \\
\hline
\end{tabular}

Hole 478

$\begin{array}{lrlllrll}1-1,15-17 & 0.15 & 3.58 & 5.933 & 2.325 & 0.539 & 1.248 & 0.555 \\ 2-3,65-67 & 7.15 & 4.45 & 8.583 & 2.523 & -0.098 & 0.675 & 0.403 \\ 2-3,80-82 & 7.30 & 5.10 & 8.250 & 2.089 & 0.183 & 0.881 & 0.468 \\ 3-1,2-4 & 13.02 & 5.35 & 8.050 & 1.767 & 0.110 & 0.857 & 0.461 \\ 3-2,70-72 & 15.02 & 5.30 & 7.616 & 1.853 & 0.304 & 0.834 & 0.454 \\ 3-3,9-11 & 16.09 & 4.50 & 7.283 & 1.949 & 0.114 & 1.086 & 0.520 \\ 3-3,56-58 & 16.56 & 2.60 & 4.516 & 1.567 & 0.594 & 1.395 & 0.582 \\ 9-5,61-63 & 76.61 & 4.35 & 8.416 & 2.621 & -0.025 & 0.696 & 0.410 \\ 15-3,77-79 & 130.77 & 4.65 & 8.050 & 2.503 & 0.250 & 0.867 & 0.464 \\ 17-3,61-63 & 149.61 & 4.20 & 6.800 & 2.048 & 0.363 & 1.006 & 0.501 \\ 28-6,36-38 & 248.86 & 4.10 & 7.466 & 2.388 & 0.113 & 0.899 & 0.473 \\ 32-1,42-44 & 269.92 & 4.30 & 7.333 & 2.363 & 0.319 & 1.281 & 0.561 \\ 32-1,110-112 & 270.60 & 1.55 & 4.683 & 2.262 & 0.210 & 0.913 & 0.477 \\ 36-1,132-134 & 308.82 & 3.70 & 5.800 & 2.186 & 0.503 & 1.411 & 0.585 \\ 39-3,57-59 & 330.07 & 4.50 & 7.966 & 2.661 & 0.290 & 0.608 & 0.378 \\ 01 e 479 & & & & & & & \\ 6-2,66-68 & 2.16 & 4.55 & 6.916 & 1.789 & 0.348 & 0.934 & 0.483 \\ 10-6,26-28 & 87.16 & 4.50 & 8.053 & 1.925 & -0.042 & 1.081 & 0.519 \\ 21-2,90-92 & 185.90 & 4.75 & 8.600 & 2.350 & 0.034 & 0.786 & 0.440 \\ 36-4,89-90 & 331.39 & 4.80 & 9.350 & 2.160 & -0.152 & 0.900 & 0.473 \\ 44-5,24-26 & 408.24 & 5.10 & 8.466 & 2.330 & 0.208 & 0.679 & 0.404\end{array}$

Hole 481

\begin{tabular}{lrrrrrrr} 
3-2, 69-71 & 11.69 & 6.15 & 9.216 & 1.968 & 0.014 & 0.690 & 0.408 \\
ole 481A & & & & & & & \\
6-1, 4-6 & 89.54 & 5.10 & 7.150 & 1.233 & -0.116 & 0.809 & 0.447 \\
$6-3,76-78$ & 93.26 & 5.00 & 7.566 & 1.994 & 0.394 & 1.315 & 0.568 \\
$6-5,116-118$ & 96.66 & 2.75 & 4.466 & 1.853 & 0.538 & 1.324 & 0.569 \\
$10-1,55-57$ & 128.05 & 4.10 & 7.416 & 2.356 & 0.293 & 0.976 & 0.494 \\
$24-2,133-135$ & 262.33 & 5.10 & 7.233 & 1.632 & 0.146 & 0.843 & 0.457 \\
$25-4,34-36$ & 274.84 & 4.85 & 6.716 & 1.530 & 0.392 & 1.055 & 0.513 \\
$27-2,40-42$ & 290.90 & 4.80 & 7.400 & 2.155 & 0.453 & 1.036 & 0.508 \\
$30-4,59-61$ & 322.59 & 4.95 & 8.450 & 1.972 & -0.065 & 1.024 & 0.506 \\
$30-6,6-8$ & 325.06 & 4.80 & 7.500 & 1.905 & 0.390 & 2.909 & 0.744 \\
\hline
\end{tabular}


Table 2. Grain-size determinations.

\begin{tabular}{|c|c|c|c|c|c|}
\hline $\begin{array}{l}\text { Core/Section } \\
\text { (interval in cm) }\end{array}$ & $\begin{array}{l}\text { Depth } \\
\text { (m) }\end{array}$ & $\begin{array}{l}\text { Sand } \\
(\%)\end{array}$ & $\begin{array}{l}\text { Silt } \\
(\%)\end{array}$ & $\begin{array}{l}\text { Clay } \\
(\%)\end{array}$ & Classification \\
\hline \multicolumn{6}{|l|}{ Hole 474} \\
\hline $1-2,45-47$ & 1.95 & 5.29 & 37.62 & 57.09 & Silty clay \\
\hline $2-2,55-57$ & 4.05 & 15.26 & 41.12 & 43.62 & Silty clay \\
\hline $3-2,50-52$ & 13.50 & 2.54 & 46.16 & 51.30 & Silty clay \\
\hline $4-2,80-82$ & 23.30 & 4.02 & 28.85 & 67.13 & Silty clay \\
\hline $5-3,47-49$ & 33.30 & 14.06 & 38.52 & 47.42 & Silty clay \\
\hline $6-4,69-71$ & 45.19 & 20.38 & 39.80 & 39.82 & Silty clay \\
\hline $7-5,110-112$ & 56.60 & 65.60 & 21.39 & 12.72 & Silty sand \\
\hline $8-2,52-54$ & 61.02 & 92.58 & 4.75 & 2.67 & Sand \\
\hline $11-2,114-116$ & 90.14 & 12.94 & 31.88 & 55.18 & Silty clay \\
\hline $12-1,40-42$ & 97.40 & 1.42 & 35.46 & 63.12 & Silty clay \\
\hline $13-1,49-51$ & 106.99 & 2.40 & 61.40 & 36.20 & Clayey silt \\
\hline $14-2,47-49$ & 117.97 & 3.77 & 41.41 & 54.82 & Silty clay \\
\hline $16-3,0-2$ & 138.00 & 8.83 & 53.83 & 37.34 & Clayey silt \\
\hline $16-3,9-11$ & 138.09 & 92.33 & 3.88 & 3.89 & Sand \\
\hline $17-5,55-57$ & 151.05 & 4.36 & 41.18 & 54.46 & Silty clay \\
\hline $17-5,77-79$ & 151.27 & 18.05 & 47.61 & 34.34 & Clayey silt \\
\hline $18-3,32-34$ & 157.32 & 12.37 & 41.43 & 46.20 & Silty clay \\
\hline $19-2,90-92$ & 165.90 & 7.66 & 39.60 & 52.74 & Silty clay \\
\hline \multicolumn{6}{|l|}{ Hole 474A } \\
\hline $1-4,68-70$ & 168.68 & 3.75 & 40.45 & 55.80 & Silty clay \\
\hline $1-4,85-87$ & 168.85 & 0.62 & 54.50 & 44.88 & Clayey silt \\
\hline $1-4,101-103$ & 169.01 & 71.28 & 23.12 & 5.60 & Silty sand \\
\hline $2-1,103-105$ & 174.03 & 21.43 & 69.16 & 9.41 & Sandy silt \\
\hline $2-1,137-139$ & 174.37 & 53.37 & 31.08 & 15.55 & Silty sand \\
\hline $3-5,35-37$ & 188.85 & 0.61 & 61.33 & 38.06 & Clayey silt \\
\hline $3-5,78-80$ & 189.28 & 1.41 & 61.10 & 37.49 & Clayey silt \\
\hline $3-5,109-111$ & 189.59 & 50.12 & 42.59 & 7.29 & Silty sand \\
\hline $3-5,137-139$ & 189.87 & 75.90 & 20.44 & 3.68 & Sand \\
\hline $4-4,76-78$ & 197.26 & 0.93 & 36.33 & 62.74 & Silty clay \\
\hline $6-1,99-101$ & 211.99 & 1.26 & 60.53 & 38.21 & Clayey silt \\
\hline $6-1,148-150$ & 212.48 & 40.90 & 42.98 & 16.12 & Sandy silt \\
\hline $6-2,11-13$ & 212.61 & 32.13 & 37.40 & 30.47 & Silt-sandy-clay \\
\hline $7-2,49-51$ & 222.49 & 6.32 & 48.38 & 45.30 & Clayey silt \\
\hline $9-3,59-60$ & 243.09 & 2.40 & 49.08 & 48.52 & Clayey silt \\
\hline $10-2,60-62$ & 251.10 & 2.10 & 66.06 & 31.84 & Clayey silt \\
\hline $12-3,62-64$ & 271.62 & 4.44 & 60.24 & 35.32 & Clayey silt \\
\hline $13-3,81-83$ & 281.31 & 0.67 & 62.87 & 36.46 & Clayey silt \\
\hline $13-4,7-9$ & 282.81 & 3.53 & 67.04 & 29.43 & Clayey silt \\
\hline $14-4,108-110$ & 283.08 & 22.48 & 60.99 & 16.53 & Sandy silt \\
\hline $13-4,116-118$ & 283.16 & 15.24 & 57.24 & 27.52 & Clayey silt \\
\hline $16-3,67-69$ & 309.67 & 4.90 & 57.95 & 37.15 & Clayey silt \\
\hline $17-4,82-84$ & 320.82 & 0.75 & 66.98 & 32.27 & Clayey silt \\
\hline $19-2,52-54$ & 329.02 & 0.27 & 53.53 & 46.20 & Clayey silt \\
\hline $21-5,79-81$ & 350.79 & 2.01 & 52.85 & 45.14 & Clayey silt \\
\hline $23-1,88-90$ & 363.88 & 33.23 & 45.42 & 21.35 & Sandy silt \\
\hline $23-4,87-89$ & 368.37 & 1.41 & 58.95 & 39.64 & Clayey silt \\
\hline $23-4,113-115$ & 368.63 & 3.98 & 67.47 & 28.55 & Clayey silt \\
\hline $25-1,62-64$ & 382.62 & 3.60 & 65.20 & 31.20 & Clayey silt \\
\hline $25-1,142-144$ & 387.92 & - & 67.00 & 33.00 & Clayey silt \\
\hline $25-5, C C, 16-18$ & 388.68 & 12.33 & 64.50 & 23.17 & Clayey silt \\
\hline $27-4,81-83$ & 406.31 & 4.30 & 78.00 & 17.70 & Silt \\
\hline $28-4,69-71$ & 414.69 & 0.84 & 48.22 & 50.94 & Silt clay \\
\hline $29-2,112-114$ & 422.62 & 1.29 & 77.52 & 21.19 & Silt \\
\hline $30-1,121-123$ & 430.71 & 2.49 & 76.87 & 20.64 & Silt \\
\hline $30-2,3-5$ & 431.03 & 13.77 & 64.29 & 21.94 & Clayey silt \\
\hline $30-2,43-45$ & 431.43 & 27.06 & 55.15 & 17.79 & Sandy silt \\
\hline $30-2,113-115$ & 432.13 & 70.24 & 21.26 & 8.73 & Silty sand \\
\hline $30-3,68-70$ & 433.18 & 1.01 & 45.41 & 53.58 & Silty clay \\
\hline $31-3,54-56$ & 442.53 & 0.24 & 75.37 & 24.39 & Silt \\
\hline $32-5,108-110$ & 455.58 & 1.49 & 79.66 & 18.85 & Silt \\
\hline $33-3,83-85$ & 460.83 & 6.07 & 63.10 & 30.83 & Clayey silt \\
\hline $36-2,104-106$ & 489.04 & 1.50 & 50.60 & 47.90 & Clayey silt \\
\hline \multicolumn{6}{|l|}{ Hole 475} \\
\hline $1-2,62-64$ & 2.12 & 3.12 & 33.58 & 63.30 & Silty clay \\
\hline $2-5,121-123$ & 12.62 & 54.50 & 18.87 & 26.73 & Clayey sand \\
\hline $3-2,74-76$ & 17.74 & 3.25 & 39.65 & 57.10 & Silty clay \\
\hline $5-4,71-73$ & 39.71 & 8.66 & 37.94 & 53.40 & Silty clay \\
\hline $6-5,23-25$ & 50.23 & 4.83 & 46.65 & 48.52 & Silty clay \\
\hline $8-5,64-66$ & 69.64 & 3.53 & 53.43 & 43.04 & Clayey silt \\
\hline
\end{tabular}

Table 2. (Continued).

\begin{tabular}{|c|c|c|c|c|c|}
\hline $\begin{array}{l}\text { Core/Section } \\
\text { (interval in cm) }\end{array}$ & $\begin{array}{l}\text { Depth } \\
\text { (m) }\end{array}$ & $\begin{array}{l}\text { Sand } \\
(\%)\end{array}$ & $\begin{array}{l}\text { Silt } \\
(\%)\end{array}$ & $\begin{array}{l}\text { Clay } \\
(\%)\end{array}$ & Classification \\
\hline \multicolumn{6}{|l|}{ Hole 475 (Cont.) } \\
\hline $\begin{array}{l}9-4,67-69 \\
11-6,16-18 \\
12-5,31-33 \\
13-4,51-53 \\
15-2,80-82 \\
15-3,77-79 \\
15-3,99-101 \\
15-4,104-106 \\
15-4,122-124 \\
15-6,65-67 \\
16-5,33-35 \\
17-2,44-46 \\
17-4,42-44\end{array}$ & \begin{tabular}{r|}
77.67 \\
99.16 \\
107.31 \\
115.51 \\
131.80 \\
133.27 \\
133.49 \\
135.04 \\
135.22 \\
137.65 \\
145.33 \\
150.44 \\
153.42
\end{tabular} & $\begin{array}{r}3.51 \\
4.30 \\
2.01 \\
1.33 \\
8.90 \\
64.60 \\
2.75 \\
6.23 \\
11.92 \\
4.02 \\
1.62 \\
14.31 \\
1.33\end{array}$ & $\begin{array}{l}36.35 \\
37.70 \\
81.39 \\
51.34 \\
48.92 \\
23.60 \\
65.75 \\
61.07 \\
67.48 \\
52.58 \\
70.92 \\
57.33 \\
68.40\end{array}$ & $\begin{array}{l}60.14 \\
58.00 \\
16.60 \\
47.33 \\
42.18 \\
11.80 \\
31.50 \\
32.70 \\
20.60 \\
43.40 \\
27.46 \\
28.36 \\
30.27\end{array}$ & $\begin{array}{l}\text { Silty clay } \\
\text { Silty clay } \\
\text { Silt } \\
\text { Clayey silt } \\
\text { Clayey silt } \\
\text { Silty sand } \\
\text { Clayey silt } \\
\text { Clayey silt } \\
\text { Clayey silt } \\
\text { Clayey silt } \\
\text { Clayey silt } \\
\text { Clayey silt } \\
\text { Clayey silt }\end{array}$ \\
\hline \multicolumn{6}{|l|}{ Hole 475B } \\
\hline $1-7,61-63$ & 9.61 & 2.52 & 26.38 & 71.10 & Silty clay \\
\hline $\begin{array}{l}1-2,48-50 \\
2-4,130-132 \\
3-3,89-91 \\
5-3,77-79 \\
6-4,71-73 \\
7-4,106-108 \\
8-2,126-128 \\
10-4,57-59 \\
11-6,99-101 \\
13-5,65-67 \\
13-3,77-79 \\
16-3,67-69 \\
17-1,85-87 \\
18-5,65-67 \\
19-1,86-88 \\
20-5,69-71 \\
21-3,28-30\end{array}$ & $\begin{array}{r}1.98 \\
14.80 \\
22.39 \\
41.27 \\
53.21 \\
61.71 \\
68.76 \\
90.07 \\
102.99 \\
120.15 \\
136.27 \\
145.67 \\
152.35 \\
167.65 \\
171.36 \\
186.69 \\
192.78\end{array}$ & $\begin{array}{r}11.43 \\
8.90 \\
2.94 \\
38.30 \\
22.09 \\
2.10 \\
2.50 \\
0.14 \\
3.33 \\
4.27 \\
58.94 \\
18.70 \\
1.54 \\
6.61 \\
10.00 \\
18.00 \\
48.95\end{array}$ & $\begin{array}{l}33.44 \\
45.50 \\
35.10 \\
27.65 \\
40.11 \\
47.30 \\
36.04 \\
39.83 \\
39.44 \\
56.98 \\
35.98 \\
35.63 \\
57.58 \\
45.45 \\
70.70 \\
42.83 \\
31.35\end{array}$ & $\begin{array}{r}55.13 \\
45.60 \\
61.96 \\
34.05 \\
37.80 \\
50.60 \\
61.46 \\
60.03 \\
57.23 \\
38.75 \\
5.08 \\
45.67 \\
40.88 \\
47.94 \\
19.30 \\
39.17 \\
19.70\end{array}$ & $\begin{array}{l}\text { Silty clay } \\
\text { Silty clay } \\
\text { Silty clay } \\
\text { Sand-clay-silt } \\
\text { Clayey silt } \\
\text { Silty clay } \\
\text { Silty clay } \\
\text { Silty clay } \\
\text { Silty clay } \\
\text { Clayey silt } \\
\text { Silty sand } \\
\text { Silty clay } \\
\text { Clayey silt } \\
\text { Silty clay } \\
\text { Clayey silt } \\
\text { Clayey silt } \\
\text { Silty sand }\end{array}$ \\
\hline $\begin{array}{l}3-2,8-10 \\
3-2,113-115 \\
4-1,81-83 \\
5-1,78-80 \\
7-1,22-24 \\
15-1,11-13 \\
16-2,24-26 \\
17-3,21-23 \\
19-2,135-137 \\
20-2,84-86\end{array}$ & \begin{tabular}{r|}
12.08 \\
13.13 \\
20.81 \\
30.28 \\
48.72 \\
105.11 \\
116.74 \\
127.71 \\
146.35 \\
155.34
\end{tabular} & $\begin{array}{r}1.21 \\
2.88 \\
1.20 \\
5.93 \\
2.33 \\
0.47 \\
0.92 \\
4.85 \\
41.21 \\
1.84\end{array}$ & $\begin{array}{l}33.31 \\
40.32 \\
35.92 \\
49.72 \\
58.60 \\
48.69 \\
57.16 \\
59.71 \\
34.76 \\
61.82\end{array}$ & $\begin{array}{l}65.48 \\
56.80 \\
62.88 \\
44.35 \\
39.07 \\
50.84 \\
41.92 \\
35.44 \\
24.03 \\
36.34\end{array}$ & $\begin{array}{l}\text { Silty clay } \\
\text { Silty clay } \\
\text { Silty clay } \\
\text { Clayey silt } \\
\text { Clayey silt } \\
\text { Silty clay } \\
\text { Clayey silt } \\
\text { Clayey silt } \\
\text { Silty sand } \\
\text { Clayey silt }\end{array}$ \\
\hline $\begin{array}{l}\text { Hole } 478 \\
1-1,15-17 \\
2-3,65-67 \\
2-3,80-82 \\
3-1,2-4 \\
3-2,70-72 \\
3-3,9-11 \\
3-3,56-58 \\
9-5,61-63 \\
15-3,77-79 \\
17-3,61-63 \\
28-6,36-38 \\
32-1,42-44 \\
32-1,110-112 \\
36-1,132-134 \\
39-3,57-59\end{array}$ & $\begin{array}{r}0.15 \\
7.15 \\
7.30 \\
13.02 \\
15.20 \\
16.09 \\
16.56 \\
76.61 \\
130.77 \\
149.61 \\
248.86 \\
269.92 \\
270.60 \\
308.82 \\
330.07\end{array}$ & $\begin{array}{r}14.12 \\
2.96 \\
2.73 \\
1.20 \\
0.93 \\
1.47 \\
51.00 \\
3.21 \\
1.36 \\
3.46 \\
4.24 \\
2.52 \\
34.98 \\
15.33 \\
1.22\end{array}$ & $\begin{array}{l}68.90 \\
39.04 \\
49.00 \\
50.86 \\
59.89 \\
59.94 \\
42.00 \\
40.40 \\
56.69 \\
67.28 \\
57.90 \\
68.60 \\
54.82 \\
68.31 \\
54.64\end{array}$ & \begin{tabular}{r|}
16.98 \\
58.00 \\
48.27 \\
47.94 \\
39.18 \\
38.59 \\
7.00 \\
56.39 \\
41.95 \\
29.26 \\
37.86 \\
28.88 \\
10.20 \\
16.36 \\
44.14
\end{tabular} & $\begin{array}{l}\text { Clayey silt } \\
\text { Silty clay } \\
\text { Clayey silt } \\
\text { Clayey silt } \\
\text { Clayey silt } \\
\text { Clayey silt } \\
\text { Silty sand } \\
\text { Silty clay } \\
\text { Clayey silt } \\
\text { Clayey silt } \\
\text { Clayey silt } \\
\text { Clayey silt } \\
\text { Sandy silt } \\
\text { Clayey silt } \\
\text { Clayey silt }\end{array}$ \\
\hline \multicolumn{6}{|l|}{ Hole 479} \\
\hline $\begin{array}{l}6-2,66-68 \\
10-6,26-28 \\
21-2,90-92 \\
36-4,89-90 \\
44-5,24-46\end{array}$ & $\begin{array}{r}2.16 \\
87.16 \\
185.90 \\
331.39 \\
408.24\end{array}$ & $\begin{array}{l}2.19 \\
2.71 \\
1.37 \\
0.25 \\
2.22\end{array}$ & $\begin{array}{l}68.18 \\
45.65 \\
41.66 \\
25.19 \\
48.34\end{array}$ & $\begin{array}{l}29.63 \\
51.64 \\
56.97 \\
74.56 \\
49.44\end{array}$ & $\begin{array}{l}\text { Clayey silt } \\
\text { Silty clay } \\
\text { Silty clay } \\
\text { Silty clay } \\
\text { Silty clay }\end{array}$ \\
\hline
\end{tabular}


Table 2. (Continued).

\begin{tabular}{lrrrrl}
\hline $\begin{array}{c}\text { Core/Section } \\
\text { (interval in cm) }\end{array}$ & $\begin{array}{c}\text { Depth } \\
(\mathrm{m})\end{array}$ & $\begin{array}{c}\text { Sand } \\
(\%)\end{array}$ & $\begin{array}{c}\text { Silt } \\
(\%)\end{array}$ & $\begin{array}{c}\text { Clay } \\
(\%)\end{array}$ & Classification \\
\hline Hole 481 & & & & & \\
3-2, 69-71 & 11.69 & 1.03 & 32.07 & 69.90 & Silty clay \\
Hole 481A & & & & & \\
6-1, 4-6 & 89.54 & 1.00 & 68.00 & 31.00 & Clayey silt \\
6-3, 76-78 & 93.26 & 1.16 & 63.00 & 35.84 & Clayey silt \\
6-5, 116-118 & 96.66 & 50.80 & 39.70 & 9.50 & Silty sand \\
10-1, 55-57 & 128.05 & 4.70 & 57.98 & 37.32 & Clayey silt \\
$24-2,133-135$ & 262.33 & 2.00 & 65.71 & 32.29 & Clayey silt \\
$25-4,34-36$ & 274.84 & 1.94 & 78.45 & 19.61 & Silt \\
$27-2,40-42$ & 290.90 & 1.03 & 70.21 & 28.76 & Clayey silt \\
30-4, 59-61 & 322.59 & 1.12 & 37.35 & 61.53 & Silty clay \\
$30-6,6-8$ & 325.06 & 0.30 & 66.63 & 33.07 & Clayey silt \\
\hline
\end{tabular}

Inclusive graphic standard deviation:

$$
\sigma_{I}=\frac{\phi 84-\phi 16}{4}+\frac{\phi 95-\phi 5}{6.6} .
$$

Skewness:

$$
S K_{I}=\frac{\phi 16+\phi 84-2 \phi 50}{2(\phi 84-\phi 16)}+\frac{\phi 5+\phi 95-2 \phi 50}{2(\phi 95-\phi 5)} .
$$

Kurtosis:

$$
K_{G}=\frac{\phi 95-\phi 5}{2.44(\phi 75-\phi 25)} .
$$

Transformed kurtosis:

$$
K_{G}^{\prime}=\frac{K_{G}}{1+K_{G}^{\prime}} .
$$

\section{RESULTS}

Triangular diagrams showing sand-silt-clay percentages for different sites are shown in Figure 2.

Table 1 lists Folk and Ward (1957) statistical parameters for all samples. Percentages of sand-silt-clay and Shepard's (1954) textural terms for individual samples are tabulated in Table 2 . Three broad textural groups are illustrated in Figure 3. Sand and silty sand sediments compose Group 1; silt, sandy silt, clayey silt, sand-silt-clay, and silty clay sediments occur in group II; and silty clays, with more than $65 \%$ of clay, constitute Group III.

\section{Hole 474}

Hole 474, $55 \mathrm{~km}$ southeast of the tip of Baja California, was continuously cored to a depth of 182.50 meters. Three sedimentary units occur in the Quaternary section.

Unit 1, 0-21 meters, is hemipelagic diatomaceous mud and ooze. Three samples were analyzed. Sediments from this unit are silty clays (Fig. 2) and occur in Group II (Fig. 3). The coarsest $5 \%$ of the distribution $(\phi 5)$ averages $4.05 \phi(60.5 \mu \mathrm{m})$. Mean size averages $7.92 \phi(4.2$ $\mu \mathrm{m}$ ). The sediments are very poorly sorted (sample
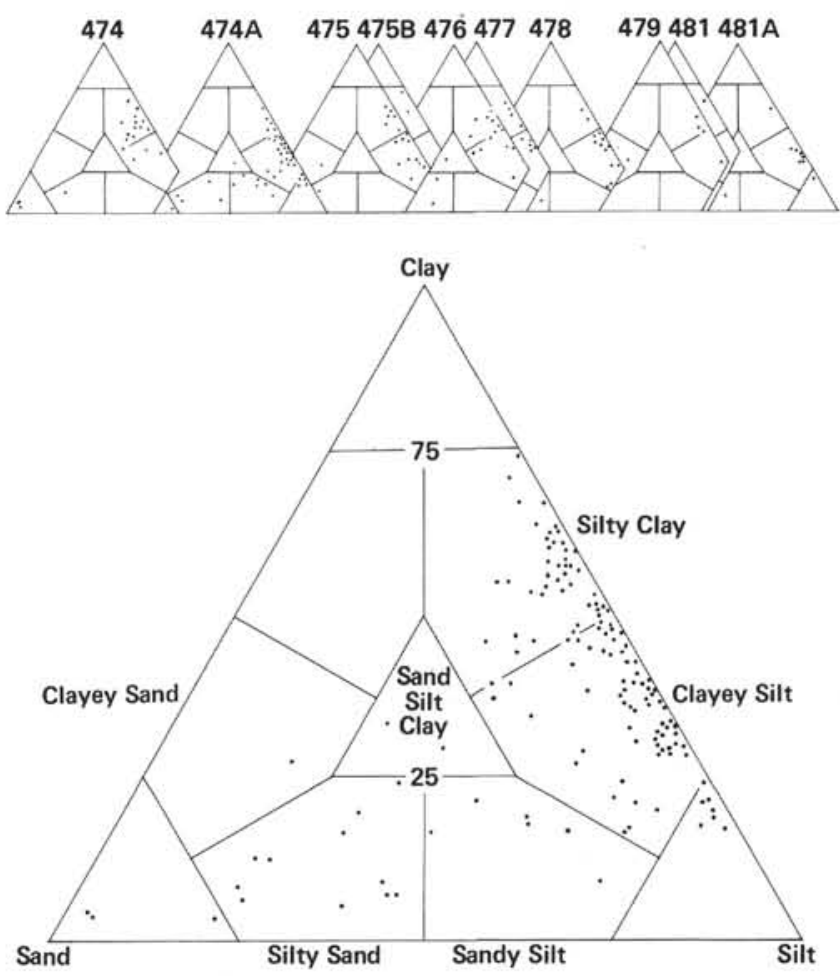

Figure 2. Sediment classification after Shepard (1954) with sand-siltclay percentages of samples from stratigraphic units at Leg 64 drilling sites.
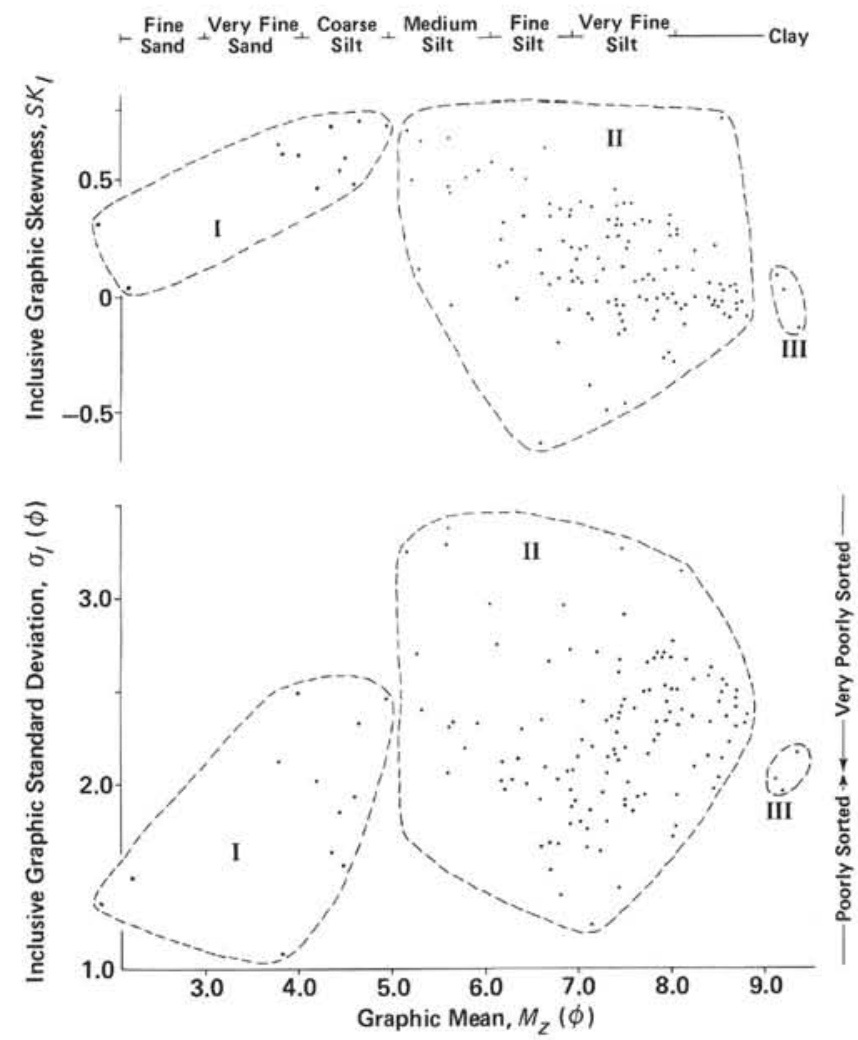

Figure 3. Textural groups of sediment from Leg 64 drilling sites, according to Inman's (1952) criteria. 
average: $\bar{x}=2.48 \phi)$ and platykurtic $(\bar{x}=0.45)$. Skewness is near symmetrical (between +0.05 and -0.07 ) and coarser toward the base of the unit.

Unit 2, 21 to 87 meters, is a sequence of cobbles, gravel, and sand, fining upward to nannofossil-rich silty clay. Five samples were analyzed. Phi 5 averages $2.42 \phi$ $(186.6 \mu \mathrm{m})$, and mean size is $5.77 \phi(18.3 \mu \mathrm{m})$. Most sediments are very poorly sorted $(\bar{x}=2.33 \phi)$, although the sand has a slightly better sorting value (Table 1). Skewness averages 0.14 (fine skewed), and transformed kurtosis is 0.50 (mesokurtic). Figure 4 illustrates vertical variations in sand-silt-clay percentage, mean size, sorting, skewness, and transformed kurtosis. Toward the base of the unit, there is an increment in the percentage of sand and a decrease in the percentage of silt and clay. Phi 5 and mean size also decrease in the same direction. Sorting becomes better, skewness is finer, and transformed kurtosis tends to be downwardly leptokurtic (Tables 1 and 2). Silty sand is the only lithologic type represented in Group I (Fig. 3); others correspond to Group II.

Unit 3, 87 to 182.5 meters, consists of deeper-water clayey silts, silty clays, nannofossil marls, and mud turbidites. Eleven samples were analyzed. Texturally, most of the samples are silty clays, and the others are clayey silts and sand (Fig. 3). The coarsest $5 \%$ average $3.47 \phi$ $(90.0 \mu \mathrm{m})$, and mean size is $7.25 \phi(6.6 \mu \mathrm{m})$. The sediments are very poorly sorted $(\bar{x}=-2.33 \phi)$, near symmetrical $(\bar{x}=-0.03)$, and mesokurtic $(\bar{x}=0.48)$. There are some vertical variations at 90,138 , and 151 meters resulting from clay or sand. Sequences of sand and clayey silt or silty clay and clayey silt may be mud turbidites with more silt and basal muddy sand occurring higher in the unit (Fig. 5). Sand tends to be coarser ( $\phi 5$ $\left.=0.00 \phi ; M_{Z}=2.18 \phi\right)$, poorly sorted $\left(\sigma_{I}=1.50 \phi\right)$, and very leptokurtic $\left(K^{\prime}{ }_{G}=0.62\right)$. The main constituents are quartz, mica (biotite), benthic and planktonic foraminifers, fish debris, and echinoid spines.

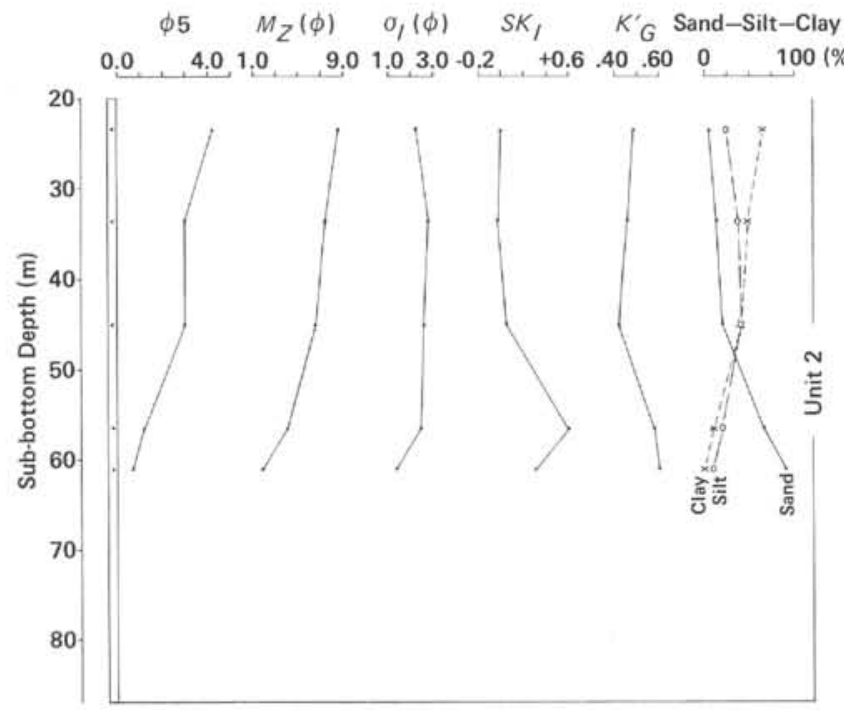

Figure 4. Vertical variations in grain-size parameters of sediment from Hole 474, Unit 2 (depth below mudline).

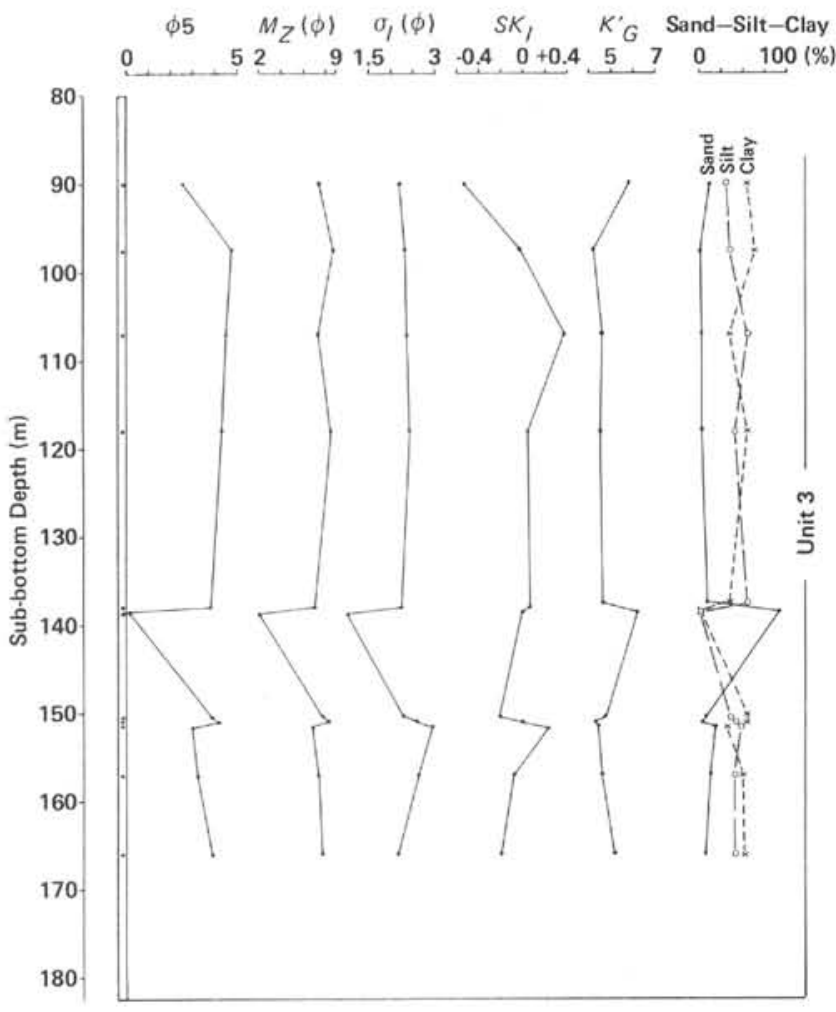

Figure 5. Vertical variations in grain-size parameters of sediment from Hole 474, Unit 3 (depth below mudline).

\section{Hole 474A}

Hole 474A was continuously cored from 163.5 meters to a total subbottom depth of 626 meters. The lowermost of the three sedimentary units of Hole 474A continued to 240 meters. Two other underlying units occur in this hole.

Unit 4 is a continuing Quaternary section of silty claystone to clayey siltstone and mudstone turbidites to 480 meters. Forty-three samples were analyzed. Texturally, this unit is extremely variable (Fig. 3). The silty claystone and clayey siltstone are clayey silt and silty clay, whereas the mudstone turbidites are silty sand, sandy silt, clayey silt, and silt. Forty-three samples were analyzed from this hole. Because of the wide variety of lithological types, average values for statistical parameters resulted in large standard deviations (Table 1). The most conspicuous textural feature of the sediments is that most of them are clayey silt and silty clay (Fig. 2, Table 2). Group I (Fig. 3) contains silty sands; all other sediments are included in Group II.

Average mean values for statistical parameters are as follows: coarsest $5 \%$ of the distribution is $3.93 \phi(65.5$ $\mu \mathrm{m})$; mean size is $6.28 \phi(12.8 \mu \mathrm{m})$; sorting is $2.13 \phi$ (very poorly sorted); skewness is 0.24 (fine skewed); and transformed kurtosis is 0.48 (mesokurtic).

There are vertical variations in the unit's grain-size parameters and sand-silt-clay percentages at 168, 174, $189,212,282,368$, and between 430 and 433 meters (Figs. 6; 7). These variations result from sequences of mud turbidites with more silt at the top and a basal or 


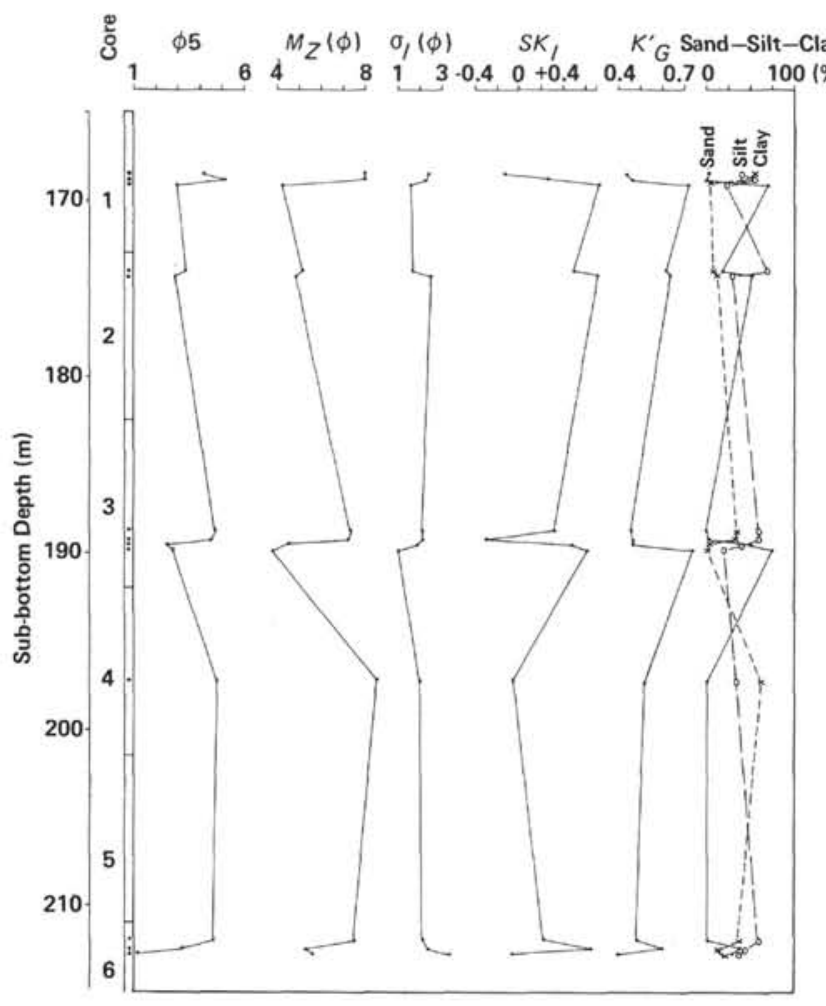

Figure 6. Vertical variations in grain-size parameters of sequences of mud turbidites in Hole 474A (depth below mudline).
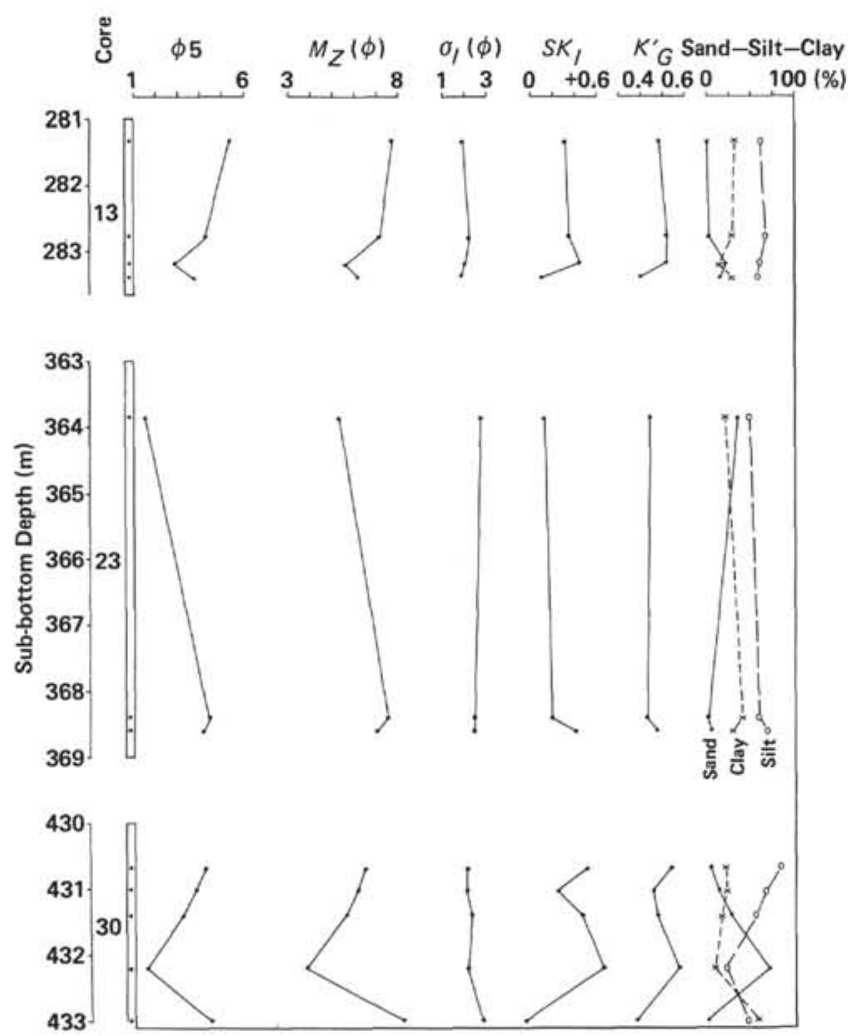

Figure 7. Vertical variations in grain-size parameters of mud turbidites in Hole 474A (depth below mudline). silty sand. These last sediments are scarce. Sand occurs at the base of a clayey silt/silty sand sequence that could be a mud turbidite; it is very fine grained, moderately sorted, and strongly fine skewed (Table 1). Sand content in silty sand sediments averages $61 \%$; phi 5 averages $2.51 \phi(178.5 \mu \mathrm{m})$, mean size is $4.42 \phi(47.0 \mu \mathrm{m})$. These sediments are very poorly sorted $(\bar{x}=2.03 \phi)$, strongly fine skewed $(\bar{x}=0.64)$, and leptokurtic $(\bar{x}=0.60)$. The coarsest $5 \%$ of the distribution and the mean size tend to be coarser with depth.

\section{Hole 475}

Hole $475(196 \mathrm{~m})$ lies in a slope basin $21 \mathrm{~km}$ southeast of the tip of Baja California. Four lithologic units occur, ranging in age from Quaternary to early Pliocene (?). Texturally, most of the sediments are silty clay and clayey silt.

Unit 1, 0 to 130 meters, is Quaternary to late Pliocene nannofossil diatomaceous silty clay to clayey silt; a subunit, from 34 to 91 meters, has few fossils. In accordance with grain-size parameters, this unit has four lithological types of sediments (Fig. 2; Table 1). The coarsest $5 \%$ of the distribution averages $4.17 \phi(56 \mu \mathrm{m})$, and mean size is $7.46 \phi(5.75 \mu \mathrm{m})$. The sediments are very poorly sorted $(\bar{x}=2.22 \phi)$, coarse skewed $(\bar{x}=$ $-0.11)$, and platykurtic $(\bar{x}=0.41)$.

Unit 2, 130 to 143 meters, is upper-to-lower Pliocene diatomaceous silty clay. We analyzed six samples. Texturally, the sediments are clayey silt and silty sand; most samples are clayey silt (Fig. 2; Table 1). Average values for statistical parameters are: $\phi 5=3.83 \phi(70 \mu \mathrm{m})$; mean size $=6.78 \phi(9.2 \mu \mathrm{m})$; sorting $=2.26 \phi$ (very poorly sorted); skewness $=0.28$ (fine skewed); and transformed kurtosis $=0.51$ (mesokurtic). Some vertical variations occur in the grain-size parameters (Fig. 8): Distinctive peaks occur between 131 and 137 meters - a result of the presence of foraminiferal sand. These fine and very fine sands are turbidites.

Unit 3,150 to 158 meters, is early Pliocene, zeolitebearing clay and dolomitic mudstone. Texturally, the two samples we analyzed from the upper part of the unit, are very poorly sorted $(\bar{x}=2.19 \phi)$, fine skewed $(\bar{x}$ $=0.218)$, clayey silt; mean-size average is $6.95 \phi(8.12$ $\mu \mathrm{m})$, and phi 5 averages $4.17 \phi(56 \mu \mathrm{m})$.

\section{Hole 475B}

This hole was drilled adjacent to Hole 475 and washed to 76 meters before we encountered basalt cobbles. Twenty meters were cored in cobbles, recovering very little. Sediments recovered were Quaternary nannofossil diatomaceous silty clay. One sample was analyzed. The sediment is a poorly sorted $\left(\sigma_{I}=1.70 \phi\right)$, coarse skewed $\left(S K_{I}=-0.29\right)$, and leptokurtic $\left(K_{G}^{\prime}=\right.$ $0.59)$, silty clay. Mean size is $3.76 \phi(74 \mu \mathrm{m})$.

\section{Hole 476}

Hole 476 lies in a small terrace on the lower continental slope southeast of the tip of Baja California. (Fig. 1). The hole was continuously cored to 294.5 meters; six lithological units occur in the hole. 


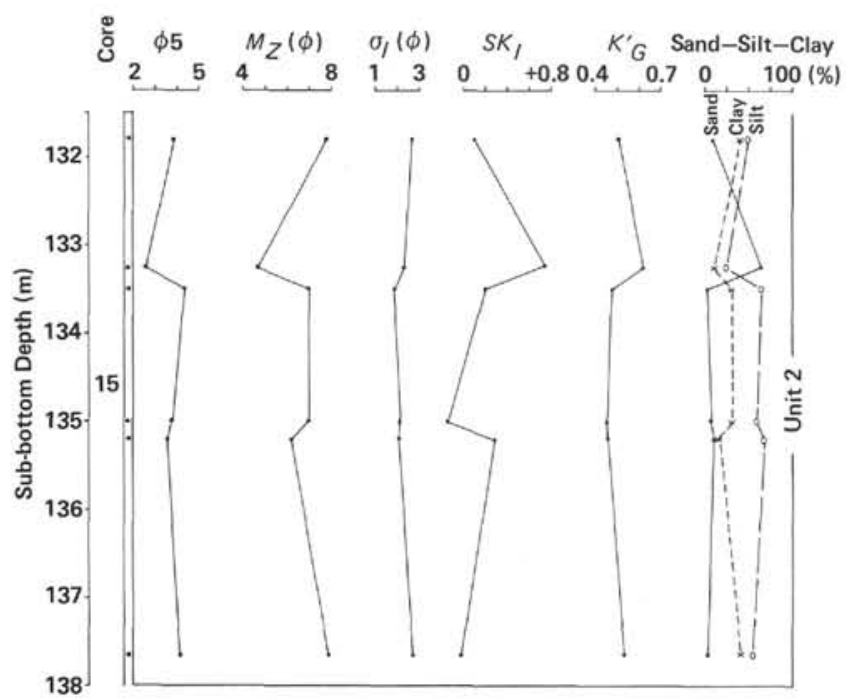

Figure 8. Vertical variations in grain-size parameters of sediment from Hole 475, Unit 2 (depth below mudline).

Unit 1 is 0.00 to 66.0 meters of Pleistocene mud and nannofossil diatomaceous ooze. Six samples were analyzed. Texturally, the unit has a variety of lithological types. Most of the sediments are silty clay (Fig. 2, Table 1). Phi 5 averages $3.42 \phi(93.5 \mu \mathrm{m})$, and mean size is 7.48 $(5.6 \mu \mathrm{m})$. Sediments are very poorly sorted $(\bar{x}=2.56 \phi)$, Skewness averages -0.03 (near symmetrical), and transformed kurtosis is platykurtic $(0.42)$. Sand decreases downward from $11.43 \%$ to $2.10 \%$ in the silty clay sediments.

Unit 2, 66.0 to 145.0 meters, is late Pliocene hemipelagic silty clay. Five samples were analyzed. Texturally, three sediments are silty clays, clayey silts, and silty sands; most samples are silty clays (Fig. 2, Table 1). The coarsest $5 \%$ averages $3.97 \phi(62 \mu \mathrm{m})$, and mean size is $7.45 \phi(5.75 \mu \mathrm{m})$; sorting is $2.16 \phi$ (very poorly sorted); skewness is -0.004 (near symmetrical), and transformed kurtosis is 0.47 (platykurtic). Vertical distribution of grain-size parameters shows peaks between 120 and 136 meters. These peaks result from the presence of clayey silts and silty sands. Sand increases slightly downhole, except in silty sand sediments, where the increment in sand percentage is abrupt (Table 2).

Unit 3, 145.0-183.0 meters, is early Miocene diatomaceous ooze with turbidites. Four samples were analyzed. Texturally, the sediments are clayey silts and silty clays. Grain-size data (Fig. 2, Tables 1 and 2) do not represent the entire unit. Average mean values are: $\phi 5$ $=3.64 \phi(80 \mu \mathrm{m})$; mean size $=7.46 \phi(5.7 \mu \mathrm{m})$. Near the top of the unit, the sediments are very poorly sorted $(3.26 \phi)$ and poorly sorted at the bottom $(1.91 \phi)$. Skewness is near symmetrical $(\bar{x}=0.01)$.

Unit $4,183.0$ to 199.0 meters, contains shallow marine claystones, glauconite sands, and barren zeolitic clays of early Pliocene to Miocene age. Grain-size data are based on only two samples (Table 1) and do not represent the entire unit. Texturally, the sediments are clayey silts and silty sands. Average mean values for statistical parameters are: Phi $5=2.37 \phi(195 \mu \mathrm{m})$; mean size $=6.0 \phi(16 \mu \mathrm{m})$. The sediments are very poorly sorted $(\bar{x}=2.98 \phi)$, strongly fine skewed $(\bar{x}=0.32)$, and mesokurtic $\left(K_{G}^{\prime}=0.48\right)$. Phi 5 and mean size tend to be coarser downward in the unit. Sand increases in the same direction (Table 2).

\section{Hole 477}

This hole, in the southern rift of the Guaymas Basin (Fig. 1), was continuously cored to a depth of 191 meters. The drilling encountered and cored a dolerite sill between 58 and 105.5 meters. Three lithological units are discernible.

Unit 1, 0.0-58.0 meters, comprises diatomaceous turbidites above the sill. Five samples were analyzed. It is mostly silty clay, although a few clayey silt layers occur (Fig. 2, Table 2). The coarsest $5 \%$ of the distribution $(\phi 5)$ averages $4.67 \phi(40.5 \mu \mathrm{m})$ and tends to be coarser in a downward direction; mean size decreases from $9.13 \phi$ $(1.82 \mu \mathrm{m})$ at the top of the unit to $7.45 \phi(5.75 \mu \mathrm{m})$ at the base and averages $4.67 \phi(39.4 \mu \mathrm{m})$. The sediments are very poorly sorted $(\bar{x}=2.22 \phi)$, near symmetrical $(\bar{x}=$ $0.00)$, and platykurtic $(\bar{x}=0.46)$, although some of the clayey silts are fine skewed and platykurtic. Others are coarse skewed and leptokurtic (Table 1).

Unit 2 is a dolerite sill.

Unit 3, 105.5 to 191.0 meters, comprises hydrothermally altered and indurated diatomaceous turbidites, ranging from clay and claystone to sandy siltstone and silty sandstone. Five samples, between 105.11 and 155.34 meters, were analyzed. This unit is texturally variable (Fig. 2). Clays and claystones are silty clays and clayey silts, whereas sandy siltstones and silty sandstones are silty sands. Because of the variety of lithological types, average values for statistical parameters display large standard deviations. Phi 5 averages $4.25 \phi$ $(52.5 \mu \mathrm{m})$, and mean size is $7.59 \phi(5.2 \mu \mathrm{m})$. The sediments are very poorly sorted $(\bar{x}=2.60 \phi)$. Skewness averages 0.24 (fine skewed), although silty sands and silty clays are near symmetrical; clayey silts are strongly fine skewed $(\bar{x}=0.44)$. All of these sediments are in Group II (Fig. 3).

\section{Hole 478}

Hole 478 lies $121 \mathrm{~km}$ northwest of the active spreading rift in the southern Guaymas Basin (Fig. 1). It was drilled continuously to a depth of 464 meters. Four lithological units occur in the section. Fourteen samples were analyzed from the units above basalt and dolerite.

Unit 1 , from 0.0 to 188.2 meters, comprises latest Pleistocene muddy diatomaceous ooze to diatomaceous mud with episodic gray sandy turbidites. The muddy diatomaceous ooze is clayey silt, whereas the diatomaceous muds are silty clay; sandy turbidites are silty sands. Most of the unit samples are clayey silts (Fig. 2). Phi 5 averages $4.40 \phi(47.4 \mu \mathrm{m})$, and mean size is $7.35 \phi$ $(6.2 \mu \mathrm{m})$. The sediments are very poorly sorted $(\bar{x}=$ $2.12 \phi)$, fine skewed $(\bar{x}=0.23)$, and mesokurtic $(\bar{x}=$ $0.48)$. Vertical variations in grain-size parameters are plotted in Figure 9. 
Silty sands are bottom sediments of turbidites that are coarser grained and better sorted than the associated clayey silt (muddy diatomaceous ooze). This sand tends to be strongly fine skewed and leptokurtic (Tables 1 and 2). Figure 9 illustrates one of the sandy turbidite sequences composed of silty sand, clayey silt, and silty clay occurring between 7 and 17 meters. This may represent two turbidite sequences.

Unit 2, from 188.2 to 260.0 meters, comprises latest Pleistocene dolomitic siltstones and diatomaceous mudstones intruded by two dolerite sills. Data on grain size (Tables 1 and 2) are based on one sample of sediment cored at 248.86 meters. It is a very poorly sorted $(2.38 \phi)$, fine-skewed $(0.11)$, and mesokurtic $(0.47)$ clayey silt. Phi 5 is $4.10 \phi(58.2 \mu \mathrm{m})$ and mean size is $7.46 \phi(5.7 \mu \mathrm{m})$.

Unit 3,260 to 342 meters, is late Pleistocene, diatomaceous silty claystones. Texturally, the sediments are clayey silts and sandy silts (Fig. 2) with the following average values: Phi $5=3.50 \phi(88 \mu \mathrm{m})$; mean size $6.45 \phi$ $(11.5 \mu \mathrm{m})$, sorting $=2.45 \phi$ (very poorly sorted); skewness $=0.22$ (fine skewed); and mesokurtosis $=0.50$. There are some vertical variations in grain-size parameters resulting from the occurrence of higher percentage $(35 \%)$, very poorly sorted $(2.26 \phi)$, fine-skewed $(0.21)$, and very platykurtic (0.47) turbiditic silty sands (Fig. 2; Tables 1 and 2).

\section{Hole 479}

Hole 479 lies on the northwest continental slope of the Guaymas Basin (Fig. 1) within the oxygen minimum and over the presumed proto-Gulf of California sediments and crust. It is located in the axis of a syncline

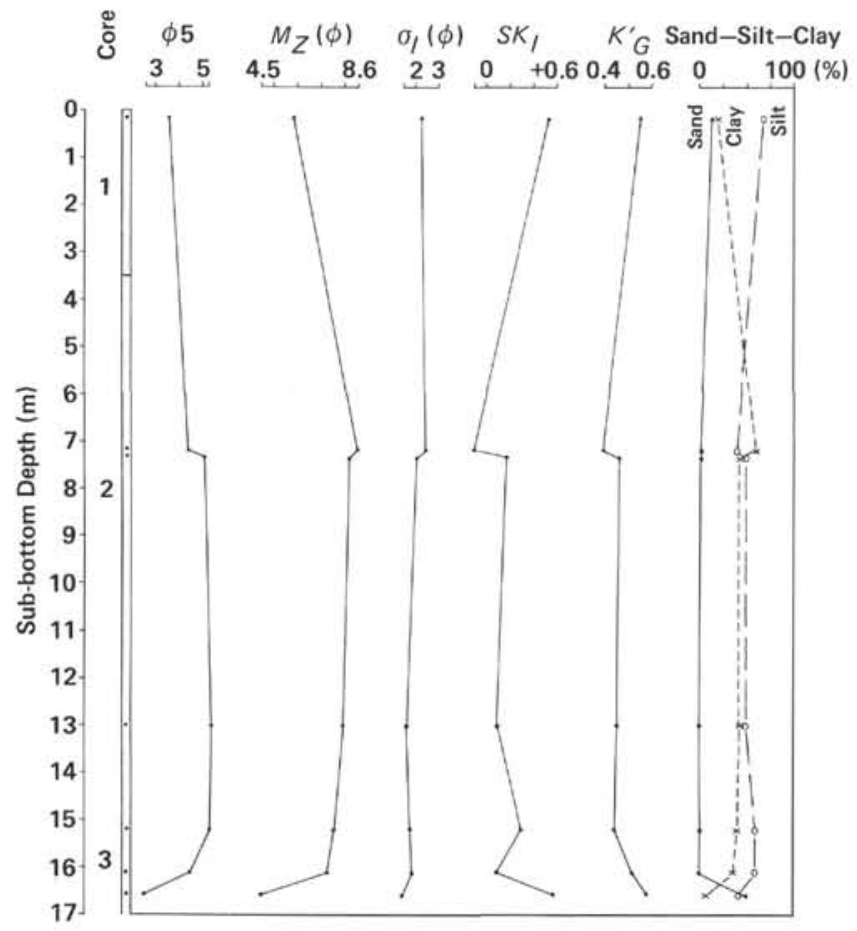

Figure 9. Vertical variations in grain-size parameters of sediment from Hole 478, Unit 3 (depth below mudline). above the scarp of the transform fault of the basin where the drilled section outcrops at the seafloor.

This hole was cored to a depth of 440 meters. The lithology of the entire section is Quaternary and the sediment is mainly muddy diatomaceous ooze with alternating sequences of varves and homogeneous, probably bioturbated, zones.

Five samples cored between 2.16 and 408.24 meters were analyzed. Texturally, most of the sediments are silty clays (Fig. 2). The coarsest $5 \%$ averages $4.74 \phi(37.2$ $\mu \mathrm{m})$, and mean size is $3.56 \phi(85 \mu \mathrm{m})$. The sediments are very poorly sorted $(\bar{x}=2.11 \phi)$, near symmetrical $(\bar{x}=$ $0.08)$, and platykurtic $(\bar{x}=0.46)$. Sand and silt percentages and clay content decrease with depth. This tendency changes at 408.24 meters, where the sand and silt percentages increase and the clay percentages diminish (Table 2).

\section{Hole 481}

Hole 481 lies near the southwestern end of the northern active spreading rift of the Guaymas Basin. This hole was drilled with the Serocki-Storms-Cameron piston corer. The lithology of the entire section is Quaternary, composed mainly of muddy diatomaceous ooze with turbidites ranging from sandy- to diatomaceous silty clay. The hole was cored only to a depth of 52.50 meters, and one sample was analyzed (Fig. 2, Tables 1,2). Texturally, the sediment corresponds with a poorly sorted $\left(\sigma_{I}=1.96 \phi\right)$, near symmetrical $\left(S K_{I}=\right.$ $0.01)$, and platykurtic $\left(K_{G}^{\prime}=0.40\right)$ silty clay.

\section{Hole 481A}

This hole, located in nearly the same position as Hole 481 , was cored from 42.0 to 384.0 meters. The sediment includes four alternating, interbedded, diatomaceous types: two distinctive turbidite types, mass flow deposits, and laminated sediments. Four sills or sill groups intrude into the soft, young sediments. Nine samples from this hole were analyzed; four were cored at 89.5-128.0 meters over the shallowest sill, and five between sills, from 262 to 325 meters.

Sediments from 89.5 to 97.0 meters are the basal part of a large, graded turbidite composed of firm clayey silt grading through sandy silt to silty sand with a thick zone of basal sand. Texturally, most of the sediments are clayey silt with lesser amounts of silty sands (Fig. 2). Mean values for statistical parameters are as follows: The coarsest $5 \%$ of the distribution averages $4.28 \phi(51$ $\mu \mathrm{m})$ and increases from $5.10 \phi(29 \mu \mathrm{m})$ at the top to 2.75 $\phi(148 \mu \mathrm{m})$ near the base. Mean size also increases from $7.15 \phi(7 \mu \mathrm{m})$ to $4.46 \phi(45.5 \mu \mathrm{m})$ in the same direction and averages $6.39 \phi(12 \mu \mathrm{m})$. The sediments are poorly sorted $(\bar{x}=1.69 \phi)$, fine skewed $(\bar{x}=0.27)$, and mesokurtic $(\bar{x}=0.52)$. Sorting is poorer in a downward direction, and skewness increases near the base of the turbidite: from coarse skewed $(-0.11)$ to strongly fine skewed $(0.54)$. Sand-silt-clay percentages are very similar in the clayey silt sediments and average 1.0, 65.0 and $33.0 \%$. Sand content increases at the base (Table 2).

Sediments cored near the contact with the first sill $(128 \mathrm{~m})$ are composed of diatomaceous mud with minor 
amounts of sand and clayey silt. One sample was analyzed and is texturally a very poorly sorted $\left(\sigma_{I}=2.35 \phi\right)$, fine skewed $\left(S K_{I}=0.29\right)$, and mesokurtic $\left(K_{G}^{\prime}=0.49\right)$ clayey silt. Phi 5 is $4.10 \phi(58.2 \mu \mathrm{m})$, and mean size is $7.41 \phi(5.9 \mu \mathrm{m})$.

\section{DISCUSSION}

Seven textural types occur in the sediment cored at eight sites during Leg 64. The types range from sand to silt and silty clay. The most conspicuous textural feature is that most of the sediments occur in the clayey silt and silty clay fields with minor amounts ranging from sand to silt or from sand to clay (Fig. 2).

The sediments range in age from late Pliocene to late Pleistocene and Recent. Grain-size results do not show any significant difference between ages (Table 3). Generally, lower Pliocene to upper Pleistocene sediments are coarser grained $(\mathrm{M}=6.23 \phi)(13.4 \mu \mathrm{m})$ and fine skewed $(\bar{x}=0.25 \phi)$. Pleistocene and upper Pliocene sediments are finer grained $(M=7.65 \phi)(5 \mu \mathrm{m})$. Pleistocene sediments are fine skewed $(\bar{x}=0.07)$ and mesokurtic $(\bar{x}=0.47)$. Upper Pliocene sediments are very poorly sorted $(\bar{x}=2.11 \phi)$, near symmetrical $(\bar{x}=$ $-0.10)$, and platykurtic $(\bar{x}=0.45)$.

A scatter plot of mean size versus sorting (Fig. 3) reveals that the best-sorted sediments have a mean diameter of nearly $4.0 \phi(62.5 \mu \mathrm{m})$ (Fig. 3, Group I); the worst-sorted sediments have mean sizes between 5.0 and $8.0 \phi(32-4 \mu \mathrm{m})$; (Fig. 3, Group III). Above $4 \phi$ and below $8.0 \phi$, sorting becomes better; mean size increases so that the best-sorted sediments in the sand and clay ranges have mean diameters between 2.0 and $4.0 \phi$ and 9.0 and 10.0ф. (Fig. 3, Groups I, and III). As Folk (1968) and Thayer et al. (1974) have pointed out, sorting depends strongly on mean size and is a function of sediment composition. Sediments displaying the best sorting values predominate in one size population (sand or silt).

Folk and Ward (1957) and Thayer et al. (1974) have shown that skewness values (greater than +0.1 or less

Table 3. Average grain-size parameters by age.

\begin{tabular}{llclcc}
\hline & \multicolumn{2}{c}{ Pleistocene } & & \multicolumn{2}{c}{ Pliocene } \\
\cline { 2 - 3 } \cline { 5 - 6 } & $\begin{array}{c}\text { Late } \\
\text { (38 samples) }\end{array}$ & $\begin{array}{c}\text { Early } \\
\text { (47 samples) }\end{array}$ & & $\begin{array}{c}\text { Late } \\
\text { (10 samples) }\end{array}$ & $\begin{array}{c}\text { Early } \\
\text { (12 samples) }\end{array}$ \\
\hline$M_{Z}$ & $7.63 \phi$ & $6.70 \phi$ & & $7.69 \phi$ & $6.70 \phi$ \\
$\sigma_{I}$ & $2.22 \phi$ & $2.22 \phi$ & & $2.12 \phi$ & $2.31 \phi$ \\
$S K_{I}$ & 0.13 & 0.19 & & -0.10 & 0.21 \\
$K_{G}^{\prime}$ & 0.50 & 0.48 & & 0.45 & 0.51 \\
\hline
\end{tabular}

than -0.1 ) departing from normality result from the subequal mixing of two normal-sized populations. Most of the sediments analyzed consist of a predominant population (fine clay) with a subordinate coarse population. This slight excess of coarse material produces the negative skewness.

Transformed kurtosis averages 0.47 (mesokurtic) for both Pliocene and Pleistocene sediments. This slight departure from normality $\left(K_{G}^{\prime}=0.50\right)$ is probably a consequence of a subequal mixing of two different-sized population (Folk and Ward, 1957; Thayer et al., 1974). In upper Pliocene sediments this results from the mixing of clay (dominant fine population) with sand or coarse silt (subordinate coarse population).

\section{ACKNOWLEDGMENTS}

I wish to thank the following persons from the Universidad $\mathrm{Na}$ cional Autónoma de México for their valuable cooperation: A. AyalaCastañares, Coordinator of Scientific Research; A. Laguarda Figueras, director of the Centro de Ciencias del Mar y Limnologia; J. C. Guerrero-Garcia; and A. Córdoba-Méndez, ex-director of the Instituto de Geologia. I would also like to thank A. Castro Del Rio, A. Pérez-Rojas, and R. López Aviles for their assistance in the laboratory work.

\section{REFERENCES}

Blatt, H., Middleton, G., and Murray, R., 1972. Origin of Sedimentary Rocks: Englewood Cliffs, NJ (Prentice-Hall).

Bode, G. W., 1974. Grain-size analyses, Leg 27. In Veevers, J. J., Heirtzler, J. R., et al., Init. Repts. DSDP, 27: Washington (U.S. Govt. Printing Office), 503-506.

Emery, K. O., 1938. Rapid method of mechanical analysis of sands. J. Sediment. Petrol., 7:105-111.

Folk, R. L., 1968. Petrology of Sedimentary Rocks: Austin, Tex. (Hemphill's Book Store).

Folk, R. L., and Ward, W. C., 1957. Brazos River bar, a study in the significance of grain size parameters. J. Sediment. Petrol., 27:327.

Griffiths, J. C., 1967. Scientific Method in Analysis of Sediments: New York (McGraw-Hill).

Inman, D. L., 1952. Measures of describing the size distribution of sediments. J. Sediment. Petrol., 22:125-145.

Irani, R. R., and Callis, C. F., 1963. Particle Size: Measurement, Interpretation, and Application: New York (John Wiley).

Krumbein, W. C., and Pettijohn, F. J., 1938. Manual of Sedimentary Petrography: New York (Appleton-Century-Crofts).

Shepard, F. P., 1954. Nomenclature based on sand-silt-clay rations. J. Sediment. Petrol., 24:151-158.

Thayer, P. A., Hostettler, J., and Smith, S., 1974. Grain-size distribution of sediment from the eastern Indian Ocean: Deep Sea Drilling Project, Leg 27. In Veevers, J. J., Heirtzler, J. R., et al., Init. Repts. DSDP, 27: Washington (U.S. Govt. Printing Office), 507-522.

Wentworth, C. K., 1922. A scale of grade and class terms for clastic sediments. J. Geol., 30:377-392. 